\title{
Robust Interpretation of User Requests for Text Retrieval in a Multimodal Environment
}

\author{
Alexandra Klein and Estela Puig-Waldmüller \\ Austrian Research Institute for \\ Artificial Intelligence \\ Schottengasse 3 \\ A-1010 Vienna, Austria \\ \{alex, stella\}@oefai.at
}

\author{
Department of Medical Cybernetics and \\ Artificial Intelligence, University of Vienna \\ Freyung 6/2 \\ A-1010 Vienna, Austria \\ harald@ai.univie.ac.at
}

\begin{abstract}
We describe a parser for robust and flexible interpretation of user utterances in a multi-modal system for web search in newspaper databases. Users can speak or type, and they can navigate and follow links using mouse clicks. Spoken or written queries may combine search expressions with browser commands and search space restrictions. In interpreting input queries, the system has to be fault-tolerant to account for spontanous speech phenomena as well as typing or speech recognition errors which often distort the meaning of the utterance and are difficult to detect and correct. Our parser integrates shallow parsing techniques with knowledge-based text retrieval to allow for robust processing and coordination of input modes. Parsing relies on a two-layered approach: typical meta-expressions like those concerning search, newspaper types and dates are identified and excluded from the search string to be sent to the search engine. The search terms which are left after preprocessing are then grouped according to co-occurrence statistics which have been derived from a newspaper corpus. These co-occurrence statistics concern typical noun phrases as they appear in newspaper texts.
\end{abstract}

\section{Introduction}

In this paper we describe a parser for robust and flexible interpretation of user utterances in a webbased multi-modal text retrieving system. The parser forms part of a system for web search in Austrian newspaper databases. In this system, users can formulate queries or navigation commands using utterances in both spontaneous spoken or written language, and they can navigate and follow links using mouse clicks. Users are completely free in formulating their utterances and in the use and combination of the input modes. Typed and spoken utterances may contain combinations of query expressions, browser commands and search space restric- tions. Users may search for texts with a specific date, in a specific newspaper or in a specific section of a newspaper. They may give complex context descriptions of the texts and they may refer to previously found texts. A dialogue manager stores actions and results from previous states and supplies information in order to construct fully specified formal queries from underspecified user requests.

In order to allow for this freedom in user behaviour, flexible processing modules are needed. For every utterance, the parser and the dialogue manager must come up with an adequate interpretation. At the same time, in interpreting the input, they have to be robust and fault-tolerant. They have to cope with typical phenomena of spontaneous speech like hesitation, correction and repetition. There may be typographical errors in written input or - even more difficult to deal with - speech recognition errors from the spoken queries. Such errors often distort the meaning of the utterance and are difficult to detect and correct.

In our interpretation component, shallow parsing techniques and knowledge-based text retrieval methods are combined to allow for robust processing and coordination of input modes. We employ a two-layered approach. The first layer serves to separate structure from content, i.e., parts of utterances referring to browser commands and search restrictions (temporal expressions, newspaper types or sections) are analyzed with a combination of keyword spotting and pattern recognition. The underlying assumption is that users will restrict themselves to a rather small vocabulary and a limited range of expressions in expressing this sort of information (this assumption is also confirmed by our Wizard-of-Oz experiments). During this process, stop words (function words and other words typically not contributing to the content of the query) are also removed. The remaining words - which are assumed to describe the search content - are then 
grouped according to co-occurrence statistics which have been derived from a newspaper corpus. While text retrieval with the help of linguistic processing has become rather common, multimodal interaction with textual databases on the web is a fairly recent application of Natural Language Processing. Experience from text retrieval shows that most information is expressed in adjective-noun, nounpreposition-noun, and noun-verb groups (Grefenstette, 1992). In our specific domain, the third type can be neglected, because verbs typically denote the action - mostly search - which is already extracted in the first layer. Thus, co-occurrence statistics consist of typical noun phrases as they appear in newspaper texts.

\section{Empirical evidence and user experiments}

In order to assess user behaviour, we carried out Wizard-of-Oz experiments (Fraser and Gilbert, 1991). Speech recognition and text retrieval were simulated. In different sessions the users interacted with a number of versions of the system: single input mode versions and versions with combinations of input modes. Their performance in terms of number of interactions as well as task completion time was measured, and their comments regarding the interface and the (simulated) system were collected in a questionnaire. Users were grouped according to previous experience with search engines and the web in general. Our results show that both, beginners and advanced users, preferred multimodal interaction over single input modes, and beginners in particular were able to speed up task completion times significantly with the help of a combination of spoken and written input with mouse clicks (Klein et al., 2001).

From these experiments, we also obtained a corpus of written and spoken utterances which were considered in the further design of the system. The queries which were posed by the users in spoken language were recorded. The recorded utterances were later read to a speech recognition system. This gave us an impression of the number and type of errors to be expected in dealing with queries in spontaneous speech.

\section{NL Text or Speech Input: Language Analysis}

Users can access articles with spoken or typed utterances. Web queries may relate to the way some particular piece of infomation is presented and what this information refers to. They may also express browser commands or a combination of browser and query commands while referring either to structure (Search for Noll in the previous newspaper) or to content (Search for Noll in the sports' section). ${ }^{1}$ Within our application web queries may relate to the way some particular piece of information is presented (e.g. the browser's history about the accessed pages), and what this information refers to (e.g. the section a search string belongs to). To successfully interpret such an utterance, one needs to analyze its structure to find out which of these command modes the utterance can be assigned to. This is done in a two-step process. First, each word is looked up in a lexicon and assigned a semantic category. Second, certain rules are applied to strings of these semantic categories. As a result, commands and search restrictions are recognized and the rest of the utterance is passed to search expression interpretation.

\subsection{Keyword Spotting and Semantic Classification}

We will now describe in more detail how the user's input is parsed within the Natural Language Interface, and structured into either search patterns - consisting of search strings, sections, dates and timeranges, that are understood by the search engine of the newspaper - or commands for the Java browser. Structure is analyzed by a flexible bottomup parser using a rule-based mechanism with simple syntactic patterns.

In the user's query input, each word of the utterance is looked up in a lexicon and - if found - assigned a corresponding semantic category. This lexicon contains a small list of semantic categories, that we consider important for the interpretation of an utterance in the domain of searching articles and browsing. The lexicon assigns semantic classes for closed categories that are:

- nouns denoting search, newspaper, section, links like "Suche" (search) or "Artikel" (article).

- nouns expressing a specific section like "Wirtschaft" (economy).

- nouns expressing a specific page like "Homepage".

- temporal expressions and temporal prepositions like "Monat" (month) and "vor" (ago).

\footnotetext{
${ }^{1}$ We will use the italic font for language expressions and the typewriter font for meta-language expressions.
} 
- expressions indicating something new like in a "neue" (new) search.

- adjectives and adverbs indicating direction in time or space, like in "letzte" (previous) search or in "letzte” (last) week.

- cardinal and ordinal numbers used in conjunction with temporal expressions and link expressions, like in "zwei" (two) years ago or when opening the "ersten" (first) link.

- adverbs and connectives indicating constraints on search mode, like "nur" (only) and "nicht" (not).

- prepositions indicating whether the request was to browse or to search, cf "zum Sport” (to the sports' section) versus "im Sport” (within the sports' section).

- stop words.

All words found within the lexicon are replaced by their corresponding semantic classes, search expressions are marked as such, and stop words are deleted.

We distinguish between semantic atoms and semantic classes: atoms by itself do not have a meaning that can be used for searching or browsing commands. They have to be joined following a given set of rules to form a semantic class. To yield such a class, rules are applied in - mostly - one to three steps. However, rules are not always necessary, a word may also be mapped onto a semantic class right away. Our lexicon has about 30 semantic atoms, from which about 40 semantic classes can be formed. Certain patterns of semantic classes which we obtain through lexical look-up can be assigned new meanings via rules. So, by composing the individual meanings, another more abstract meaning is defined. This compositional approach to interpretation is supported by the layered approach. The result of this process is a list of chunks ${ }^{2}$, where stress is laid on the content words. The advantage of concentrating on chunks is - especially within German, a language with a relatively free word order - that the order in which chunks occur is much more flexible than the order of words within chunks. This approach might be too shallow for a deeper semantic analysis, but is sufficient for our needs. So, e.g.

\footnotetext{
${ }^{2}$ According to Abney (1991) a chunk is defined in terms of major heads where a major head is any content word that does not appear between a function word $f$ and the content word $f$ selects, OR a pronoun selected by a preposition. [...] The typical chunk consists of a single content word surrounded by a constellation of function words, matching a fixed template.
}

"letzte" (last) plus a time expression would together yield the new meaning date_- $1 \mathrm{w}$. To overcome ambiguities and avoid potential rule conflicts, rules spanning larger chunks have a higher priority and are thus preferred, such that "zurïck zum Sport" (back to sports) would win over "zurück" (back). If no rules can be applied to a semantic class, it will be ignored in the final interpretation.

Summing up the process of the structure analysis, the partial analyses are stored, a sequence of partial analyses from the set of rules is chosen, and then combined to yield larger structures.

\subsection{Search String Filter: Extraction of Adjective-Noun Pairs}

In the next step, the content of the query must be analyzed in more detail. In this chapter we will explain how content analysis is done in our application.

From a corpus of Austrian newspaper texts, adjective-noun- and adjective-proper-name pairs were extracted and counted. These pairs were stored and consulted in query interpretation. Since the texts are tagged manually, the lists of adjectives and nouns/proper names contain a considerable number of errors. Therefore it is necessary to use large amounts of text; it may even be useful to eventually introduce a threshold so that only adjectivenoun/proper-name pairs which appear more than once or a certain number of times are considered. This of course can not prevent systematic tagging errors.

A robust stemming algorithm maps all adjectivenoun/proper-name pairs to an approximate 'stem', thus eliminating flectional forms which result in morphological variation which is typical for the German language. For the purpose of creating a repository of co-occurrence pairs, we do not care about proper stemming. Rather, it is our aim to map various inflectional forms onto one base form.

Spelling variations, numbers etc. are smoothed as far as it is possible in automatic processing. For example, ordinal numbers which are labelled as adjectives are reduced to a placeholder for numbers.

Whenever a word is encountered in processing which can be considered an adjective, it is kept. Whenever the following word may be a noun or a proper name, it is checked whether the adjectivenoun/proper-name combination is contained in the repository of adjective-noun/proper-name combinations which has previously been extracted from a corpus. If the adjective-noun/proper-name combination is found, it is passed on to the search engine 
as a query. Whenever the combination has not occurred in the corpus, only the noun or proper name is considered a key word.

Again, inflectional variations as well as different spellings etc. are mapped onto base forms as far as possible. The same stemming algorithm is used which was employed in creating the repository of adjective-noun/proper name pairs. The robust (and rough) stemming and categorization algorithms produce a certain amount of mistakes in the lists of pairs as well as in the mapping process, but taking into account larger text corpora evens out these problems as more text is processed.

Our approach distinguishes noun phrases which have a record of co-occurrence from noun phrases which may be spontaneous expressions or modifications or even errors created by users. For example, the phrase "europäische Staaten" (European countries) would be retained while "beteiligte Staaten" (participating countries) would be reduced to the noun. Some adjectives used in search expressions serve to qualify the global search expression rather than the noun or proper name in quesion. For example, a search for yesterday's speech would only yield articles from the day after a speech, not about the speech in general.

\section{Action History: Integration of the knowledge sources}

Multimodal dialogue requires a unified interpretation of the involved knowledge sources, all input modes have to be considered. The information transmitted needs to be interpreted within discourse context including previous user actions, possibly with data coming from other input modes.

After the analysis of the user utterance has been performed in the pattern-matching and the searchword-extraction modules, the computed meaning of the utterance has to been interpreted in the context of the discourse sitiuation. This concerns mostly the history of previous queries. Here, it is important to consult previous queries in all possible input modes (spoken, typed, mouse clicks). Therefore, a record of the action history is kept and consulted. All typed, written and spoken actions are assigned an entry in the action history where the main parameters and their values are collected.

With this contextual information, the meaning of the user's utterance as the sum of the results of the component analyses is computed in the global discourse context. Underspecified queries can be in- terpreted in the discourse contexts, and parameters are filled. Thus, the results are combined into one unambiguous command line.

A powerful interaction control is necessary in order to recognize the user's intent by comparing it to what the system knows about the addressed entities and their relation to each other as well as to the data which are accessible at the specific moment in the interaction. The interface language between the language analysis module and the controller consists of a fixed set of parameters, which are assigned appropriate values:

- DIRECTION

the direction for browsing (forward, backward)

- SECTION the section in the newspaper (politics, sports, ...)

- SEARCHSTRING the string which has to be searched by the newspaper search engine

- DATE the date when the article to be searched has appeared (also intervals)

- ZEITUNG (NEWSPAPER) the newspaper which is supposed to be searched

- OPENLINK

the link in a document which should be followed in the browser

- OPENURL the URL which is supposed to be opened by the browser

The outcome - or left-hand side - of a rule-based simplification can be divided into three command types:

- Simple Search Command, New Search Command: E.g. "Suche nach Camilleri im Kulturressort" (Search for Camilleri in the cultural section) or "Neue Suche beginnen mit Krimis" (Start a new search on thrillers).

- Complex Search Command: Search using the Action history. E.g. "Suche nach Christie im letzten Ressort" (Search for Christie in the previous section).

- Simple History Browsing Commands: Normal Browsing using the Accessed Page History. E.g. "Zur nächsten Seite gehen" (Go to the next page).

- Complex History Browsing Commands: Browse using the Action history. E.g. "Geh zum letzten Ressort" (Go to the last ressort) or "Zurück zur Suche mit Montalbano gehen" (Go back to the search containing Montalbano). 
- WWW Browsing: E.g. "Geh zum heutigen Sportbereich" (Go to today's sport section), "den Standard lesen" (read the Standard) or "Geh zur Homepage" (Go home).

- Opening Link Command: E.g. "den ersten Artikel öffnen" (Open the first article)

The action history browsing command refers to the timeline and the point of reference of a browsing but also of a search command. For instance, take an utterance, where someone wants to search for a topic but within a context that was defined in the previous search. For our application, we would first have to locate the user's point of reference and then execute her search command. If there is no given reference, we assume by default that a new time point is created in our time line.

One such command could look like this: the utterance "Ich suche etwas über Highsmith im letzten Ressort" (I am looking for something about Highsmith within the previous section) would be mapped to: DIRECTION 0, SECTION $x$ (where $\mathrm{x}$ is the section of the action with index -1), SEARCHSTRING Highsmith, TIME nil, ZEITUNG nil. We are not moving in the timeline, instead we are adding a new search action, thus the direction is zero. Anyway, the controller has to look up the action history to fill the value of the section. The values of all empty parameters will be filled with the values of the last actions, so in our example, these parameters have not been explicitly filled and remain empty (nil).

\section{Result: Translating into Http Request or Browser Command}

After the command has been processed by the control module, it is either executed by the Java browser or translated into a GET method through an Http request to the newspaper's archive database. The resulting articles are displayed in the Java browser, another search can be started by the user.

\section{Conclusion}

We have presented an interpretation component for natural language user input in a web-based multimodal text retrieval system. By applying wellknown and simple methods from shallow parsing and knowledge-based text retrieval and integrating them in a novel way we have succeeded in creating a robust, flexible and efficient parser for our application.

An important feature is the distinction between those parts of utterances relating to structure and those relating to content. This is achieved by taking advantage of the fact that only a limited vocabulary and set of expressions are used for the former. This allows us to employ simple rule-based techniques for their interpretation. The identification of the content on the other hand is done with the help of a co-occurrence repository, at the moment consisting of adjective-noun/proper name pairs. In the future we will have to investigate whether search results can be improved by inserting other combinations, like noun-preposition-noun triples.

\section{Acknowledgements}

This work was supported by the Austrian Science Fund (FWF) under project number P-13704. Financial support for ÖFAI is provided by the Austrian Federal Ministry of Education, Science and Culture.

\section{References}

Steven Abney. 1991. Parsing by chunks. In Robert Berwick, Steven Abney, and Carol Tenny, editors, Principle-Based Parsing, Tübingen (Germany). Kluwer Academic Publishers.

Norman M. Fraser and G. Nigel Gilbert. 1991. Simulating speech systems. Computer Speech and Language, 5(1):81-99.

Gregory Grefenstette. 1992. Use of Syntactic Context to Produce Term Association Lists for Text Retrieval. In N.J. Belkin, P. Ingwersen, and A.M. Pejtersen, editors, Proceedings of the 15th Annual International ACM SIGIR Conference on Research and Development in Information Retrieval, pages 89-97, Copenhagen: Denmark. ACM Press.

Alexandra Klein, Ingrid Schwank, Michel Généreux, and Harald Trost. 2001. Evaluating Multimodal Input Modes in a Wizard-of-Oz Study for the Domain of Web Search. In Ann Blandford, Jean Vanderdonckt, and Phil Gray, editors, People and Computer XV - Interaction without Frontiers: Joint Proceedings of HCI 2001 and IHM 2001, pages 475-483. Springer: London, September. 